\title{
Positional changes of pericentromeric heterochromatin and nucleoli in postmitotic Purkinje cells during murine cerebellum development
}

\author{
I. Solovei, ${ }^{\text {N N. Grandi, }}{ }^{\text {R. Knoth, }}$ b. Volk, ${ }^{\text {b }}$ and T. Cremer ${ }^{a}$ \\ a Department of Biology II, Human Genetics, Ludwig Maximilians University (LMU), Munich; \\ b Department of Neuropathology, Neurocentre, University of Freiburg, Freiburg (Germany)
}

\begin{abstract}
Previous studies revealed changes of pericentromeric heterochromatin arrangements in postmitotic Purkinje cells (PCs) during postnatal development in the mouse cerebellum (Manuelidis, 1985; Martou and De Boni, 2000). Here, we performed vibratome sections of mouse cerebellum (vermis) at P0 (day of birth), at various stages of the postnatal development (P2-P21), as well as in very young (P28) and 17months-old adults. FISH was carried out on these sections with major mouse satellite DNA in combination with immunostaining of the nucleolar protein B23 (nucleophosmin). Laser confocal microscopy, 3D reconstructions and quantitative image analysis were employed to describe changes in the number and topology of chromocenters and nucleoli. At all stages of postnatal PC development heterochromatin clusters were typically associated either with nucleoli or with the nuclear periphery, while non-associated clusters were rare $(<1 \%$ at $\mathrm{P} 0$ to $\mathrm{P} 21$ and about $3 \%$ in adult stages). At P0, about $2-4$ nucleoli and 7-8 pericentromeric heterochromatin clusters were variably located within PC nuclei. The relative volume of heterochromatin clusters associated with the nucleoli (about $50 \%$ ) was rough-
\end{abstract}

ly equal to the volume of clusters associated with the nuclear periphery. Positional changes of both nucleoli and centromeres towards the nuclear center occurred between P0 and P6. At P6 the average number of chromocenters per PC nucleus had decreased to about five. In agreement with previous studies, one or occasionally two nucleoli were noted at the nuclear center surrounded by major perinucleolar heterochromatin clusters. The relative volume of these perinucleolar clusters increased to about $84 \%$, while the volume of clusters in the nuclear periphery decreased to about $15 \%$. At subsequent postnatal stages, the arrangement of most pericentromeric heterochromatin around a central nucleolus was maintained. In adult animals, however, we observed a partial redistribution of heterochromatin towards the nuclear periphery. The average total number of pericentromeric heterochromatin signals increased again to about ten. The volume of heterochromatin associated with the nuclear periphery roughly doubled (30\%), while the volume of the perinucleolar heterochromatin decreased correspondingly.

Copyright $\odot 2004$ S. Karger AG, Basel
Purkinje cells (PCs) are known as the efferent elements of the cerebellar cortex. They form a monolayer located at the boundary between the molecular layer at the cerebellar periphery and the granular layer towards the cerebellar interior. In

Supported by a grant from the Deutsche Forschungsgemeinschaft to T.C. (Cr 59/ 20-3).

Received 14 October 2003; manuscript accepted 15 December 2003.

Request reprints from Irina Solovei, Department of Biology II

Humangenetik, Ludwig Maximilians University (LMU)

Richard Wagner Str. 10, 80333 München (Germany)

telephone: +49-89-2180-6713; fax: +49-89-2180-6719

e-mail: Irina.Solovei@lrz.uni-muenchen.de adult animals PCs show a characteristic cellular and nuclear morphology. In pioneering non-isotopic in situ hybridization studies, Laura Manuelidis employed mouse satellite DNA as a probe to pericentromeric heterochromatin of different mouse neuronal cell types (Manuelidis, 1984a, b). In these studies Manuelidis described cell-type-specific patterns of satellite DNA clusters in neuronal cell nuclei, including nuclei from PCs. In newly born mice, PCs are already in a postmitotic state but not yet terminally differentiated. In detailed follow-up studies of PC nuclei during postnatal development starting a week after mouse birth, Manuelidis found that the nuclear morphology begins to assume its adult shape between day 7 to day 11: a central nucleolus became recognizable and nuclei showed 2 to 4 heterochromatin clusters, similar to nuclei of adult PCs. \begin{tabular}{lll}
\hline KARGER & $\begin{array}{l}\text { Fax +41 61306 } 1234 \\
\text { E-mail karger@karger.ch } \\
\text { www.karger.com }\end{array}$ & ( 2004 S. Karger AG, Basel \\
$0301-0171 / 04 / 1054-0302 \$ 21.00 / 0$
\end{tabular}
Accessible online at: www. karger.com/cgr 
At this stage, some clusters were adherent to the nucleolus, while others were still located at the nuclear periphery - in contrast to adult Purkinje neurons, which showed a rather exclusive positioning of heterochromatin around a single centrally located nucleolus (Manuelidis, 1985). These observations strongly indicate a migration of pericentromeric heterochromatin during terminal differentiation away from the nuclear membrane and towards the nuclear center. During the same period synapses of the dendrite tree commence. A similar architecture of adult PC nuclei with major clusters of constitutive heterochromatin around a large central nucleolus was also observed in other species, such as guinea pigs and hamsters (Manuelidis, 1984a, b). The correlation between these profound changes in the nuclear architecture and the formation of a functional dendrite tree has raised considerable interest in a possible role of a dynamic higher order nuclear architecture in the cell-type-specific functioning of PCs and other neuronal cell types. The evolutionary conservation of cell-type-specific patterns, despite differences in centromere DNA sequences, further emphasizes a functional role that is not yet understood.

In subsequent studies, Martou and De Boni (2000) confirmed a striking kinetochore movement in PC nuclei between the day of birth (P0) and day 29 after birth (P29) when the mouse becomes a young adult with a fully developed cerebellum. These authors performed immunostaining of cerebellar sections with CREST antiserum at different postnatal stages. Following light optical sectioning of PCs, they counted the total number of kinetochore signals per nucleus and determined the percentages of centromeres associated with the nucleolus and the nuclear periphery, respectively, or showing an intermediate position, remote from either nucleoli or the nuclear periphery. During postnatal differentiation of PCs, they found changes in the aggregational state of kinetochores, including a decrease of the signal numbers at early stages of postnatal development and an increase during later stages. The fraction of kinetochore signals found in the nuclear periphery decreased during terminal PC differentiation, while the fraction associated with the centrally located nucleolus increased.

In the present study we used state-of-the-art technology including laser confocal microscopy, 3D reconstructions, and quantitative image analysis of mouse PC nuclei to describe changes in the number and topology of centromere clusters and nucleoli at defined days of postnatal development (P0, P2, P3, $\mathrm{P} 4, \mathrm{P} 6, \mathrm{P} 14, \mathrm{P} 21)$, in very young adults (P28), as well as in fully matured 17-month-old adults.

\section{Materials and methods}

\section{Murine cerebellum sections}

Adult $\mathrm{C} 57 \mathrm{Bl} / 6$ mice (P28 and 17 months old) and mice at different postnatal developmental stages (P6, P14, P21) were anaesthetized with isoflurane (Abbott, Wiesbaden, Germany) and by intraperitoneal administration of a mixture of ketamine (100 mg/kg; Parke Davis, Berlin, Germany) and xylazine (Rompun, 5 mg/kg; Bayer, Leverkusen, Germany). Mice were transcardially perfused with Ringer's solution and then with $4 \%$ formaldehyde in PBS (pH 7.2) for $10 \mathrm{~min}$, followed by immersion fixation of the removed brains in the same fixative overnight. Brains of P0, P2, P3, and P4 mice were removed unfixed immediately after decapitation and fixed by immersion into $4 \%$ formaldehyde overnight. Tissues were embedded in $4 \%$ agarose and sections of $60 \mu \mathrm{m}$ thickness through the vermis of the cerebellum were cut coronally on a Vibratome (VT1000S; Leica, Heidelberg, Germany). Sections were kept in PBS with $0.1 \%$ sodium azide at $4^{\circ} \mathrm{C}$ until use. Purkinje cells (PCs) from any part of the vermis were studied for stages P0 to P3; at older stages, the second and third folia of the vermis could be clearly recognized and PCs from only these two folia were analyzed.

\section{Immunostaining}

For immunostaining individual floating sections were permeabilized with $0.5 \%$ Triton $\mathrm{X}-100$ for $1 \mathrm{~h}$ and then sequentially incubated with primary and secondary antibodies diluted in PBS with $0.1 \%$ Triton X-100 and $1 \%$ BSA at $37^{\circ} \mathrm{C}$. As a PC marker, calbindin was detected with a mouse anticalbindin antibody (1:1000; monoclonal, IgG; Swant, Bellinzona, Italy) for $12 \mathrm{~h}$. Nucleoli were detected by protein B23 (nucleophosmin/NPM) staining using a mouse anti-B23 antibody (1:500, monoclonal; IgG, Sigma) for $12 \mathrm{~h}$. In both cases, incubation with primary antibody was followed by incubation with goat anti-mouse antibody conjugated to Alexa $488(1: 500 ; \operatorname{IgG}[\mathrm{H}+\mathrm{L}]$, $\mathrm{F}\left(\mathrm{ab}^{\prime}\right)_{2}$; Molecular Probes, Eugene, OR, USA) for $3 \mathrm{~h}$. Nuclei were counterstained with DAPI $(0.5 \mu \mathrm{g} / \mathrm{ml})$ and TO-PRO-3 $(1 \mu \mathrm{M})$ in PBS. Sections were mounted on microscopic slides with antifade Vectashield (Vector).

Fluorescence in situ hybridization (FISH)

As a marker of all centromere regions in mouse cells, a mouse major satellite probe repeat was generated by PCR with 5'-GCG AGA AAA CTG AAA ATC AC and 5'-TCA AGT CGT CAA GTG GAT G primers and murine genomic DNA as a template. The probe was labeled with TAMRAdUTP (Perkin Elmer) or Cy3-dUTP (Amersham Biosciences) by nick translation and dissolved in hybridization mixture (50\% formamide, $10 \%$ dextran sulfate, $1 \times \mathrm{SSC}$ ) at a concentration of $10 \mathrm{ng} / \mu \mathrm{l}$.

Floating vibratome sections were used in experiments performed at P0, P6 and P28, where FISH was combined with immunostaining of the nucleolus marker B23 (nucleophosmin). Sections were permeabilized with $1 \mathrm{M} \mathrm{NaSCN}$ (at $37^{\circ} \mathrm{C}$ for $45 \mathrm{~min}$ ), digested with $0.06 \mathrm{mg} / \mu \mathrm{l}$ pepsin (in $0.01 \mathrm{~N} \mathrm{HCl}$ at $37^{\circ} \mathrm{C}$ for $15 \mathrm{~min}$ ) and equilibrated in $50 \%$ formamide $/ 2 \times \mathrm{SSC}$ (at $37^{\circ} \mathrm{C}$ for $30 \mathrm{~min}$ ). Thereafter a single section was placed on a microscope slide, covered with probe in hybridization mixture, mounted under a glass chamber, and sealed with rubber cement. The chamber consisted of a $12 \times 12 \mathrm{~mm}$ coverslip with glued glass strips with a height of $0.7 \mathrm{~mm}$. Typically, 20-40 $\mu$ l of hybridization mixture was sufficient to fill the chamber. Tissue DNA and probe DNA were denatured simultaneously at $85^{\circ} \mathrm{C}$ on a hot block for $5 \mathrm{~min}$. Hybridization was performed at $37^{\circ} \mathrm{C}$ for $3-5$ days in humid dark boxes. After hybridization sections were washed in $2 \times \mathrm{SSC}$ at $37^{\circ} \mathrm{C}(3 \times 10 \mathrm{~min})$, blocked in PBS with $2 \%$ BSA and $0.1 \%$ Triton X-100 for $15 \mathrm{~min}$, and stained with anti-B23 antibody (see above). Finally, sections were counterstained with DAPI and TO-PRO-3, and mounted in antifade (Vectashield).

It was difficult to handle floating mouse cerebellum sections of early postnatal stages due to their small size and fragility. To overcome this problem and ensure the comparability of quantitative evaluation for early and late postnatal stages, we performed FISH to dehydrated sections attached to SuperFrost slides in all experiments which did not require anti-B23 immunostaining. Sections were placed in a drop of water on the slide, air-dried at room temperature for $30 \mathrm{~min}$ and dehydrated through an ethanol series $(30$, 50, 70, 90, and 100\%). Permeabilization and pepsin digestion of attached sections were performed as described above. Then sections were again dehydrated, air-dried, mounted in hybridization mixture under the glass chamber, and sealed with rubber cement. While the hybridization efficiency obtained with floating and attached sections was similar, the morphology of the floating sections, which were never dried during the whole procedure (in contrast to attached sections) was superior.

\section{Microscopy}

Sections were viewed using an epifluorescence microscope (Axioplan II; Zeiss, Germany) equipped with Plan Apo $20 \times$, 40×, 63× and $100 \times / 1.4$ oil immersion objectives and filter sets for DAPI, Alexa 488, Cy3, and TOPRO-3. Digital images of metaphase spreads and DAPI-stained sections were acquired by a CCD-camera (CoolView; Photonic Science, UK) using CytoVision software (Applied Imaging, UK). The 8-bit grayscale single fluorochrome images were overlaid to an RGB image assigning a false color to each fluorochrome.

Series of optical sections through PC nuclei were collected using a Leica TCS SP confocal system equipped with a Plan Apo $63 \times / 1.3$ NA oil immer- 

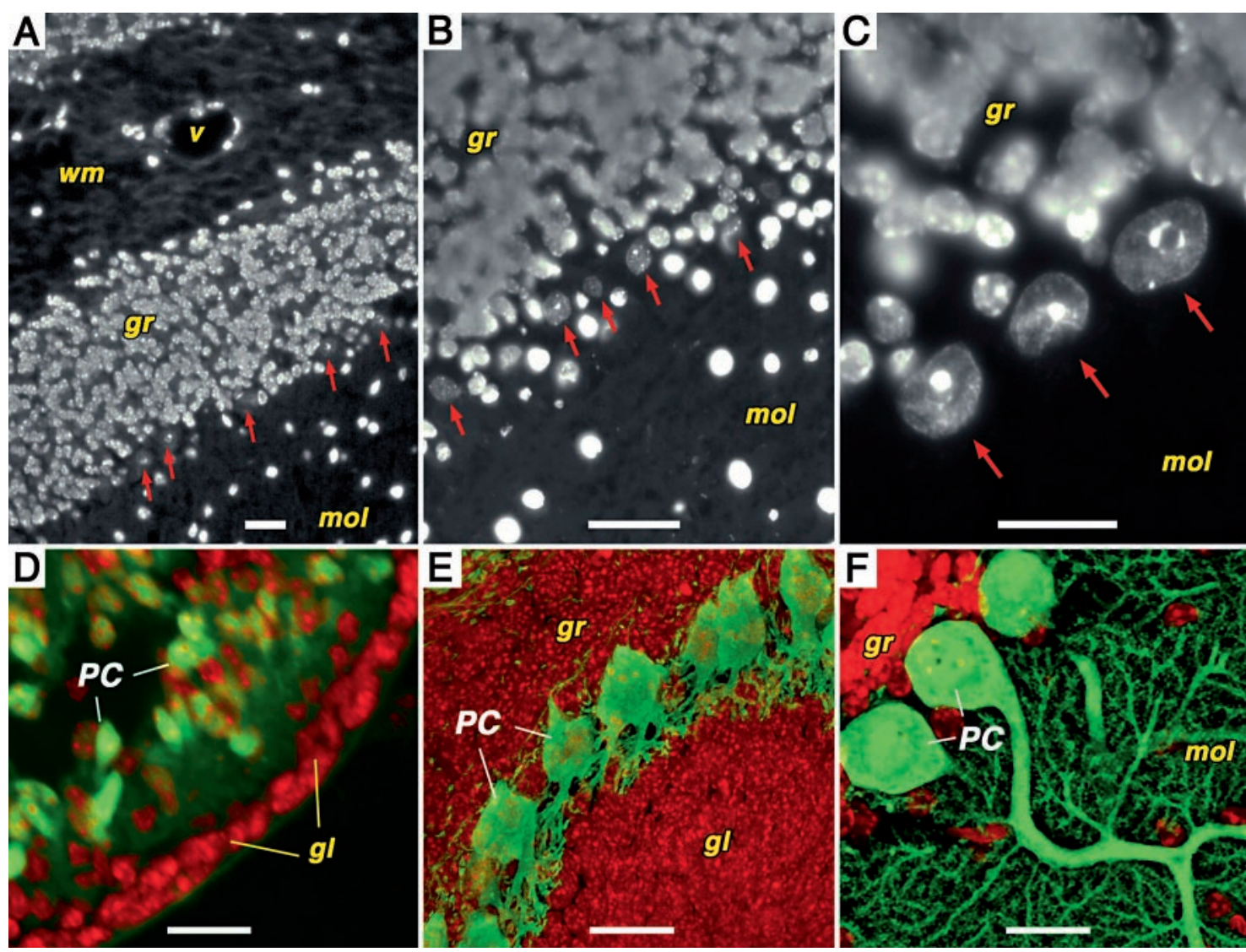

Fig. 1. Vibratom sections through vermis of cerebellum of an adult mouse. (A-C) Localization and morphology of Purkinje cell nuclei in DAPI-stained sections at increasing microscopic magnification. PC nuclei are the biggest in the tissue and reveal weakly stained nucleoplasm with brightly stained chromocenters. gr: granular layer; mol: molecular layer; wm: white matter, $v$ : blood vessel. (D-F) Maximum intensity projections of confocal serial sections of PCs stained with anti-calbindin (green) and TO-PRO-3 (red). (D) PCs at P0 have a multilayer arrangement and are situated beneath the germinal layer $(g l)$ from which granular cells originate. (E) PCs at P6 form a monolayer between granular cells $(g r)$ and germinal layer $(g l)$. Note the beginning of the dendrite tree growth at this stage. (F) PCs at P28 with a completely developed dendrite tree protruding into the molecular layer (mol). Scale bars are $20 \mu \mathrm{m}$ in $\mathbf{C}-\mathbf{F}$, and $50 \mu \mathrm{m}$ in $\mathbf{A}$ and $\mathbf{B}$.

sion objective. Fluorochromes were visualized using an argon laser with excitation wavelengths of $488 \mathrm{~nm}$ (for Alexa 488) and $514 \mathrm{~nm}$ (for Cy3 and TAMRA), and a helium-neon laser with the excitation wavelength of $633 \mathrm{~nm}$ (for TO-PRO-3). For each optical section, images were collected sequentially for two or three fluorochromes. Stacks of 8-bit grayscale images were obtained with axial distances of $\sim 300 \mathrm{~nm}$ between optical sections and pixel sizes of $80 \mathrm{~nm}$. For a typical PC nucleus from adult mouse a complete image stack comprised about 35 sections.

Image processing and quantitative assessment of chromocenter arrangement in Purkinje cells

Galleries of RGB confocal images were assembled using ImageJ and Adobe Photoshop programs. Three-dimensional reconstruction of nuclei and their chromocenters was performed by surface rendering of image stacks using Amira 2.3 TGS (http://www.amiravis.com). This program was also used for volume measurements of nuclei after nuclear segmentation. ImageJ (http://rsb.info.nih.gov/ij) was employed for signal counting in pseudo-colored image stacks and volume measurements of chromocenters.

For a quantitative evaluation of the distribution of nucleoli and chromocenters on mid sections through Purkinje cell nuclei, the RRD (Relative Radius Distribution) computer program was used (for detailed description see Cremer et al., 2001). Briefly, the program works as follows: (1) The gravity center of a given nucleus and its borders are determined on the basis of the nuclear counterstain signal. (2) Borders of chromocenters or nucleoli are determined by thresholding of the fluorescence signals in the respective color channels. (3) The nuclear radius in any direction from the nuclear center (defined as the intensity gravity center of the DNA counterstain) to the segmented nuclear edge is normalized to $100 \%$ and the nuclear space is divided into 25 shells of equal thickness (each covering $4 \%$ of the total radius). In this way the distribution of pericentromeric heterochromatin or nucleoli (estimated as fluorescence signal intensity) was measured and expressed as a function of the relative distances of each shell from the nuclear center. For signals with a random radial distribution we expect the same distribution as for the counterstained nuclear DNA. Significant deviations from the counterstain curve indicate a non-random radial distribution. To compare relative positions of targeted structures, we calculated the average relative radius (ARR). ARR presents the mean value of the distribution of all distances from all voxels representing a signal to the gravity center of the counterstained nucleus.

\section{Results}

Figures 1A and B show the typical location of PCs in fluorescent images from sections through adult cerebellar vermis following nuclear DNA staining with DAPI. Higher magnification 
A

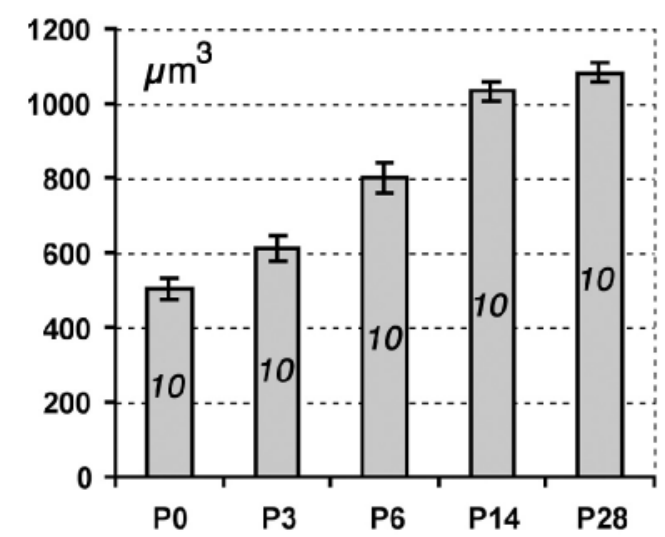

B

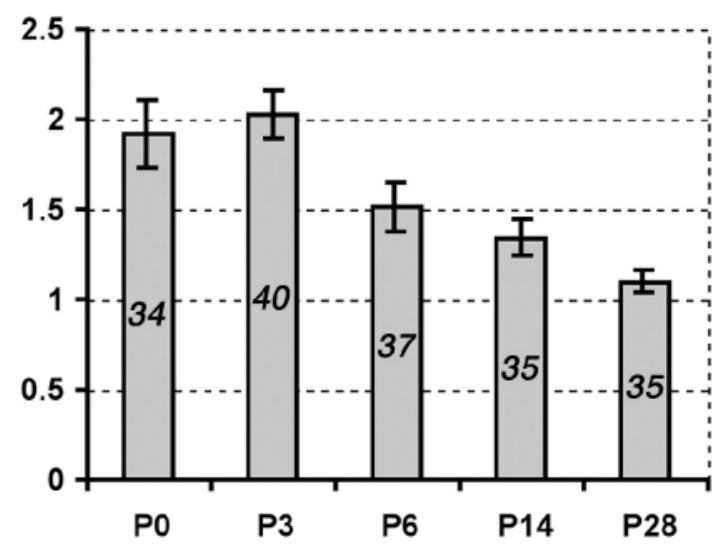

Fig. 2. Changes of the nuclear volume $(\mathbf{A})$ and the number of nucleoli $(\mathbf{B})$ in PC nuclei as a function of postnatal cerebellum development. Bars represent standard errors of the mean; numbers of analyzed nuclei per stage are shown inside the columns.

(Fig. 1C) reveals the large nuclei and the typical nuclear staining pattern of PCs in more detail. Most of the volume of PC nuclei stains weakly with DAPI (due to a strong chromatin decondensation), while heterochromatin forms $2-3$ bright globules closely associated with the nucleoli situated in the center of the nucleus. Nucleoli could be readily identified in PC nuclei stained with DAPI and/or TO-PRO-3 as round non-stained areas of the nucleoplasm encircled by more intensely stained chromatin (see e.g. Fig. 3B-E). In preliminary experiments we tested whether PCs could be unequivocally identified based on their location and their nuclear staining pattern. For this purpose we combined DNA staining with DAPI and TO-PRO-3 with immunofluorescent staining of calbindin, a calcium-binding protein which provides a highly specific marker protein for the detection of PCs in the cerebellum (Legrand et al., 1983; Nordquist et al., 1988; Fenili and De Boni, 2003) (Fig. 1D-F). We first viewed cerebellar cortex sections in the DAPI or TOPRO-3 channel and identified potential PCs; thereafter we switched to the calbindin channel and confirmed the presence of calbindin in all these cells. We conclude that it is sufficient to employ DNA staining alone for the identification of PCs. In contrast to DAPI, TO-PRO-3 has its emission in the far-red part of the spectrum and could be detected by the confocal microscope available for the present experiments. Accordingly, we employed DAPI to screen tissue sections by conventional epifluorescence microscopy and TO-PRO-3 to perform light optical sectioning. The positioning of the $\mathrm{PC}$ monolayer and the morphology of PC nuclei typical for adult mice were already manifested at $\mathrm{P} 6$ of postnatal mouse development. At the earlier stages, PCs were still represented by a multilayer (P0P2, Fig. 1D). Granular cells, initially localized outward to PCs in the outer granular layer (also called germinal layer), had migrated inward and could be observed on either side of PCs (P3-P4). Nevertheless, additional immunostaining with calbindin revealed that DAPI/TO-PRO-3 stained PC nuclei could be distinguished from nuclei of other cell types solely on the basis of their location and size.
We measured the size of $\mathrm{PC}$ nuclei at five stages of the cerebellum development: P0, P3, P6, P14, and P28. Image stacks were obtained from ten TO-PRO-3 stained nuclei at each developmental stage, their 3D reconstructions were performed and volumes were calculated using Amira software. Intense growth of dendrite trees between stages P3 and P14 (Fig. 1D-F) was accompanied by a significant growth of nuclear volume, from 500 to $1000 \mu \mathrm{m}^{3}$ (Fig. 2A). Similar changes in volume of PC nuclei (from 400 to about $1000 \mu \mathrm{m}^{3}$ ) were described for developing rat cerebellum between P6 and adult stages (Lentz and Lapham, 1970). Two, three or even four small nucleoli were typically found in the cerebellum of newborn mice (see e.g. Fig. 5A). During postnatal development of the cerebellum the average number of nucleoli per nucleus decreased to 1-2 at P6 (Fig. 2B). PCs of adult animals usually had a single, very large central nucleolus (see e.g. Figs. 3B, E, 5C).

The mouse major satellite repeat was used to label the pericentromeric heterochromatin of all mouse chromosomes (Fig. 3A). PC nuclei in the second and the third folia of the cerebellar vermis were scanned using confocal microscope and light optical image stacks were arranged as galleries. Figure 3B exemplifies a typical distribution of pericentromeric heterochromatin in a PC nucleus. In murine interphase nuclei, centromere heterochromatin often fuses to chromocenters of variable sizes. Therefore, the observed number of major satellite signals in PC nuclei was always below the diploid chromosome number $(2 n=40)$. Noteworthy, one or two heterochromatin globules stained with DAPI/TO-PRO-3 were not recognized by major satellite probe indicating that they did not contain major satellite but other DNA. These globules were typically observed at the nuclear periphery (not shown) or adjacent to the nucleolus (arrows on Fig. 3B, D).

To trace the dynamics of chromocenter formation, about 30 nuclei were scanned at each stage of postnatal development (P0, P2, P3, P4, P6, P14, P21) and for adult animals (P28 and 17 months old). Data for P28 and 17-month-old animals were pooled, since they were not significantly different (individual 

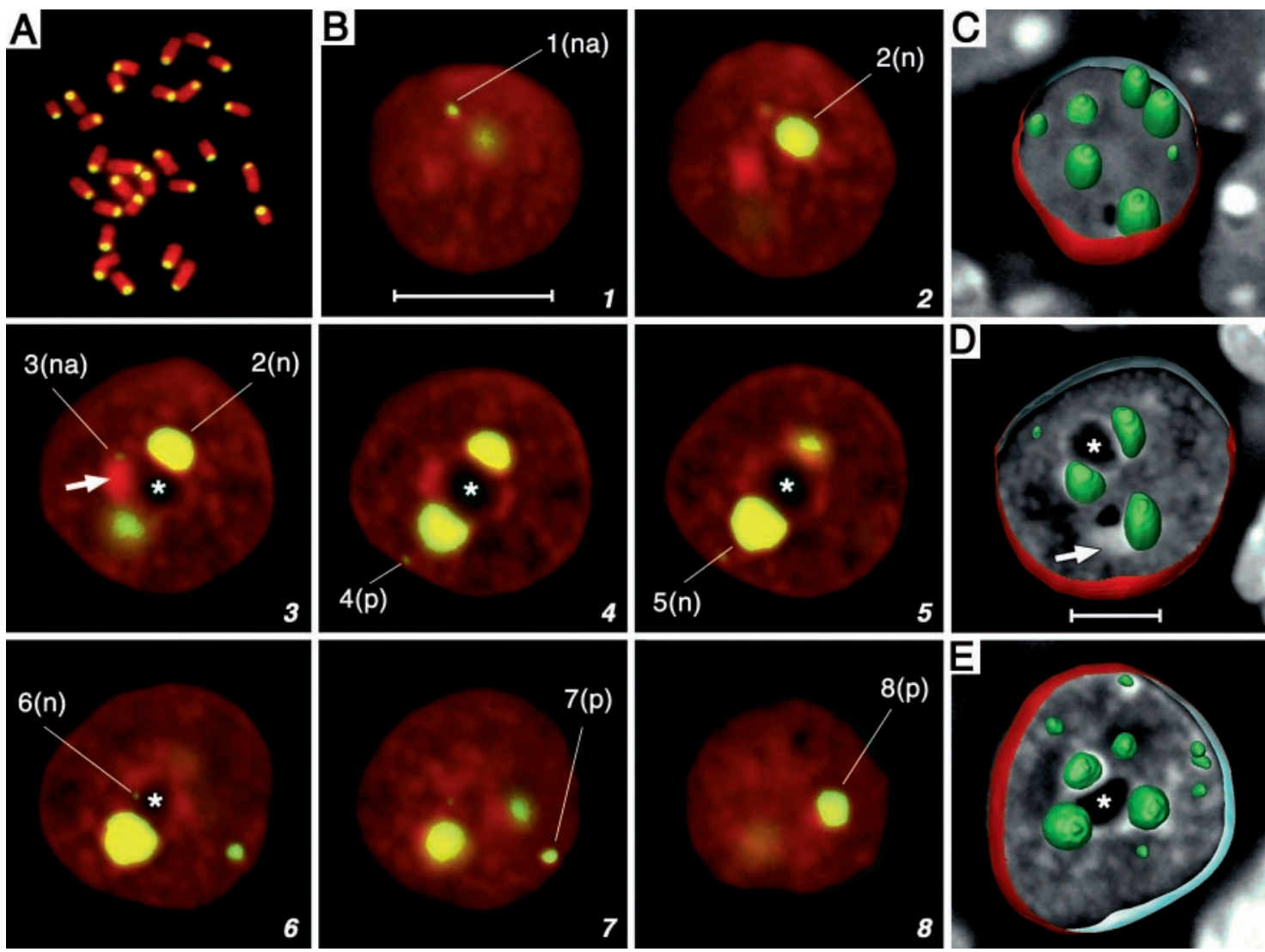

Fig. 3. (A) Metaphase spread from a mouse fibroblast after FISH with the mouse major satellite (m.s.) DNA probe and DAPI counterstain (red, false color) demonstrates staining of pericentromeric heterochromatin (green). Due to intense staining of pericentromeric heterochromatin with both DNA stain and m.s. probe, pericentromeric regions have yellow color. (B) Gallery of confocal sections (numbered from 1 to 8 ) through the mid-part of a PC nucleus at P21 stained with TO-PRO-3 (red) after 3D FISH with the m.s. probe (green). Every third section is shown. Eight heterochromatin signals are numbered in order of their appearance and marked with respect to their intranuclear location as adjacent to the nuclear periphery $(p)$ or to the nucleolus (n), or to none of these structures (non-adjacent: na). The arrow in section 3 denotes a big heterochromatin cluster adjacent to the nucleolus $(*)$, but not stained by the m.s. probe. Due to intense staining of pericentromeric heterochromatin with both DNA stain (red) and m.s. probe (green), pericentromeric regions on $\mathbf{A}$ and $\mathbf{B}$ appear yellow. (C-E) 3D reconstruction of $\mathrm{PCs}$ at P0 (C), P6 (D), and P28 (E). Heterochromatin signals (green) are shown together with a partial reconstruction of the nuclear border (red). An optical mid nuclear section of TO-PRO-3 counterstain is shown in gray color. Scale bar in $\mathbf{B}$ is $10 \mu \mathrm{m}$, scale bar in $\mathbf{D}$ is $5 \mu \mathrm{m}$ and applied to $\mathbf{C}$ and $\mathbf{E}$. data not shown). For the same reason, data for early stages of development (P2, P3, and P4) were also pooled into one category, $\mathrm{P} 2-4$. In newborn mice (P0), the average total number of chromocenters per PC nucleus was $7.6 \pm 0.4$. At P6, the number had decreased to about $5 \pm 0.3$ and increased again at the subsequent stages (P14 and P21). The highest average number of $10.4 \pm 0.6$ chromocenters per PC nucleus was observed in adults (Fig. 4A). With regard to their location in the nucleus, individual chromocenters were classified to three categories (Fig. 3B): adjacent to the nuclear periphery (p), adjacent to the nucleoli (n), and non-adjacent to either periphery or nucleoli (na). The peripheral signals (p) revealed the same developmental dynamics as described above for the total number of signals (Fig. 4B, black columns), i.e. the average number of peripherally located chromocenters was reduced from about five at P0 to two at P6 and then increased again from P6 to adults. The number of nucleoli-adjacent signals increased from P0 to P2 1 (Fig. 4B, gray columns). The number of the non-adja- cent signals was very low at all stages, although a slight increase was apparent from P0 to adult stages (Fig. 4B, white columns).

Pericentromeric heterochromatin signals significantly varied in size and position depending on the developmental stage and number of chromosomes participating in the formation of chromocenters. Chromocenters in PC nuclei of P0-P4 animals were rather uniform in size (about $1.5 \mu \mathrm{m}$, Fig. 3C) and were observed both at the nuclear periphery and adjacent to nucleoli. In contrast, in P6 to adult animals, most pericentromeric heterochromatin was organized into 2-3 large chromocenters (on average about $3 \mu \mathrm{m}$ in diameter) adjacent to 1 or 2 centrally located nucleoli. Peripherally located chromocenters in adult animals were much smaller (on average about $1 \mu \mathrm{m}$ in diameter). The non-adjacent signals were typically small and apparently formed by pericentromeric regions of very few or single chromosomes (Fig. 3B).

Our counts of the numbers of chromocenters at different stages of postnatal development did not take into account their 
A

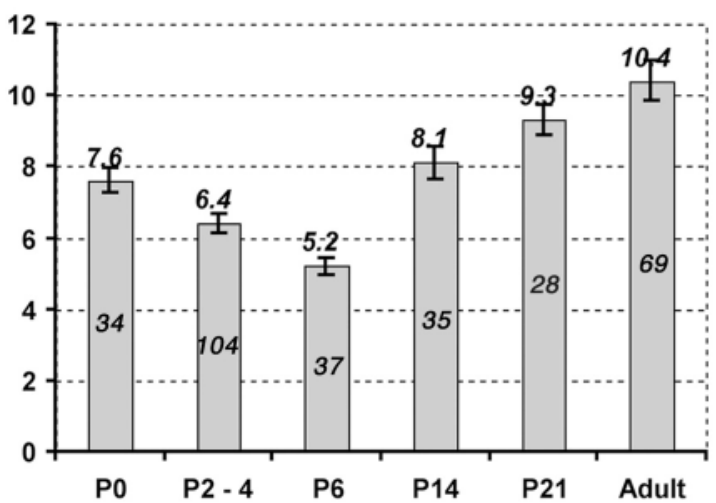

B

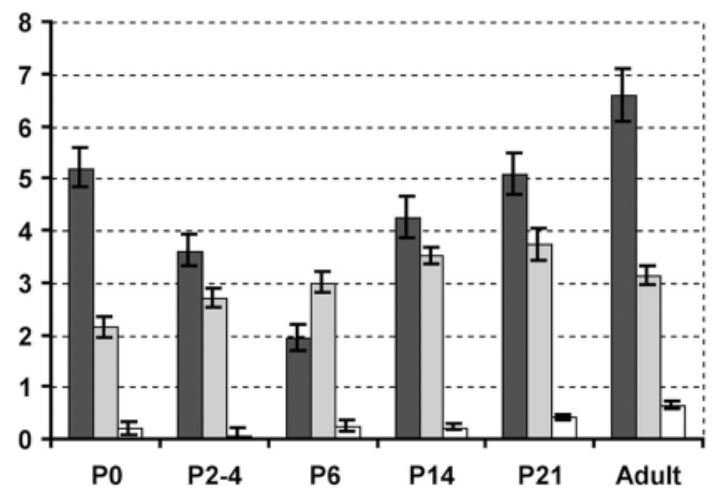

C

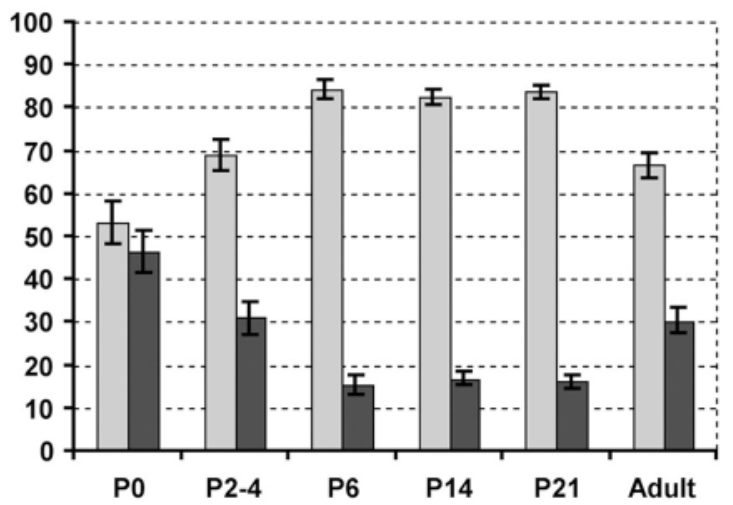

D

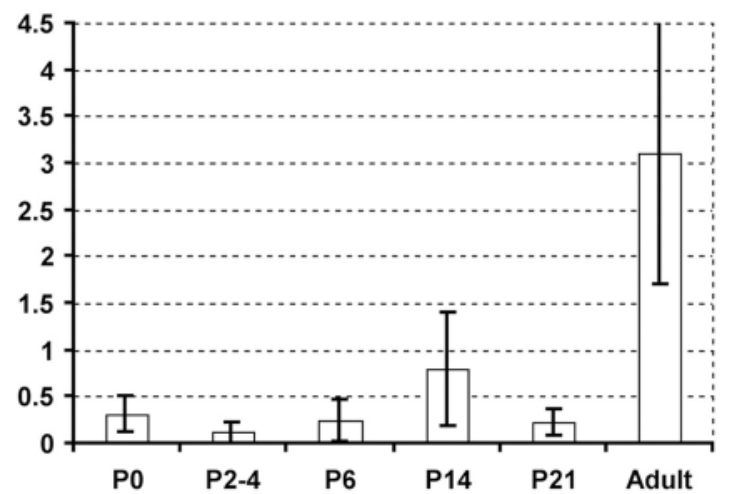

Fig. 4. Quantitative analysis of $P C$ nuclei at different stages of postnatal development. (A) Average total number of pericentromeric signals. (B) Average number of signals adjacent to the nuclear periphery (black columns), to the nucleoli (gray columns), and of non-adjacent signals (white columns). (C) Average volumes of signals associated with the nuclear periphery (black columns) or with nucleoli (gray columns) given as per cent to the total signal volume. (D) Volumes of non-adjacent signals given as per cent to the total signal volume. Bars represent standard errors of the mean. The numbers of nuclei analyzed per stage are shown inside columns in $\mathbf{A}$ and applied to the rest of the graphs.

pronounced size variations. This prompted us to attempt volume measurements of individual chromocenters. Figures $4 \mathrm{C}$ and $\mathrm{D}$ show the relative volume of the three signal categories defined above ( $p, n, n a)$ as percentage of the total volume of pericentromeric heterochromatin signals occupied in each nucleus. Taking into account that voxel numbers strongly depend on the chosen threshold that separates a signal from background, absolute volume measurements are unreliable, if not impossible, while relative volume measurements may provide more useful information. These data indicate that at $\mathrm{P} 0$, roughly half of the volume of pericentromeric heterochromatin was associated with nucleoli (Fig. 4C, gray columns), while the other half was associated with the nuclear periphery (Fig. 4C, black columns). At P2-4 the fraction of the volume of nucleolus associated chromocenters increased to $70 \%$ and at P6 to $80 \%$, while only about $15 \%$ of the volume remained associated with the nuclear periphery. This distribution was stably retained at subsequent stages (P14 and P21). In PC nuclei from adult animals the fraction of the volume of nucleolus associated heterochromatin decreased slightly (to about $65 \%$ ), while the amount of peripheral heterochromatin increased from about 16 to $30 \%$. Relative volumes of clusters which were neither associated with nucleoli nor with the nuclear envelope were very small at all developmental stages: less than 1\% for P0-P21 and about 3\% for adult animals (Fig. 4D).

The data described above support a correlation in space and time between the dynamics of nucleolus and chromocenter positioning during the postmitotic differentiation of PC nuclei. To study the distribution of pericentromeric heterochromatin and nucleoli in PC nuclei in more detail, we combined FISH with the major satellite probe with anti-B23 immunostaining for three developmental stages showing major differences in the distribution of pericentromeric heterochromatin, P0, P6, and P28. The dense packaging of cells in the cerebellum tissue made it difficult to resolve upper and lower borders of PC nuclei due to the limited Z-resolution of the laser confocal microscope, which under practical conditions is slightly less than $1 \mu \mathrm{m}$. Since borders of nuclei could be much better defined in the XY plane (due to the better resolution of about $300 \mathrm{~nm}$ ), we used mid optical nuclear sections through PC 
A

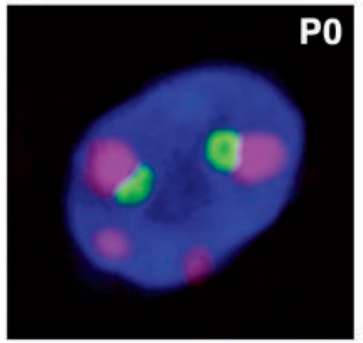

B

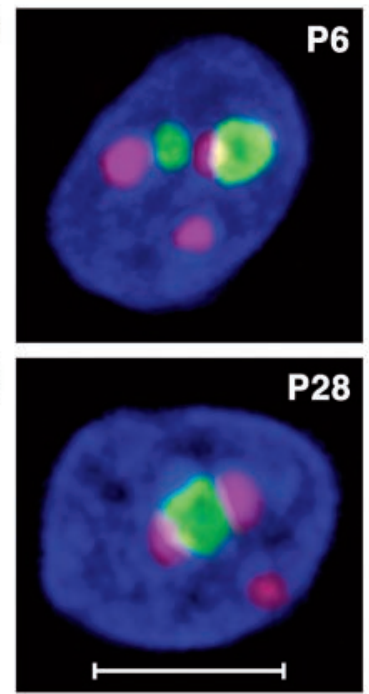

Fig. 5. (A-C) Representative mid sections through PC nuclei at P0 (A), P6 (B), P28 (C) after 3D FISH with mouse major satellite probe (red), immunostaining of the nucleolar marker B23 (green), and DNA counterstain with TO-PRO-3 (blue). Bar $=10 \mu \mathrm{m}$. (D-F) Radial distribution of B23 (green curves), pericentromeric heterochromatin (red curves) and TO-PRO-3-stained DNA (blue lines) in mid sections of PC nuclei at $\mathrm{P} 0$, P6 and P28. (G) Comparison of average relative radii (ARR) of nucleoli (green), chromocenters (red), and for the TO-PRO-3 counterstain (blue). (H) Scheme of major positional changes of the number and location of nucleoli (green) and pericentromeric heterochromatin clusters at $\mathrm{P} 0, \mathrm{P} 6$ and P28. Arrows in the nuclear scheme at P0 indicate the inward direction of movements and clustering of both nucleoli and heterochromatin that occurs between $\mathrm{P} 0$ and $\mathrm{P} 6$. As a result a large central nucleolus is typically present in PC nuclei at P6. Arrows indicate the outward movements of a part of the perinucleolar heterochromatin between P6 and P28. In addition to the de-clustering of the perinucleolar heterochromatin fraction traveling back to the nuclear periphery, it is possible that heterochromatin permanently located at the nuclear periphery splits and forms two or more smaller clusters. For simplicity, the scheme shows a nuclear projection where peripheral clusters are only drawn at the $\mathrm{X}, \mathrm{Y}$-nuclear rim, in reality peripheral clusters can be associated anywhere at the 3D nuclear envelope, most likely with the lamina.
G

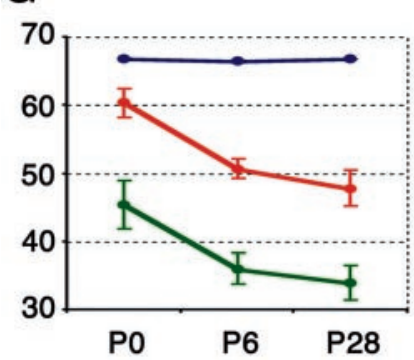

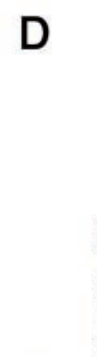
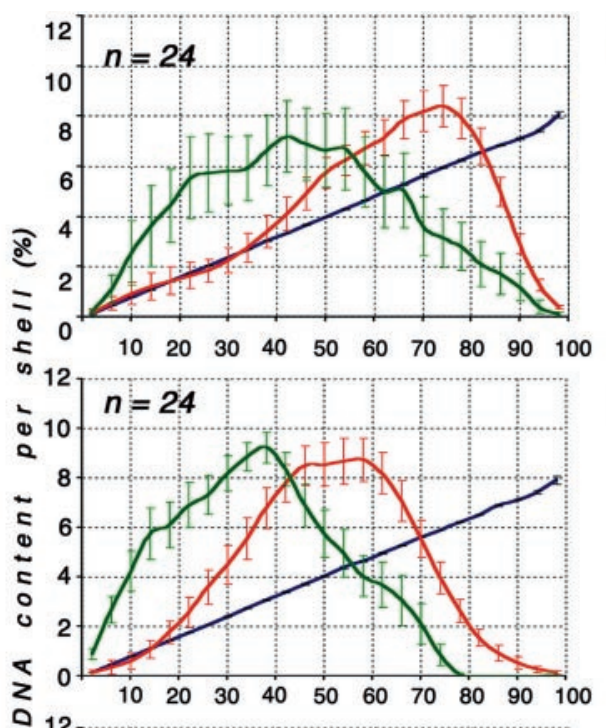

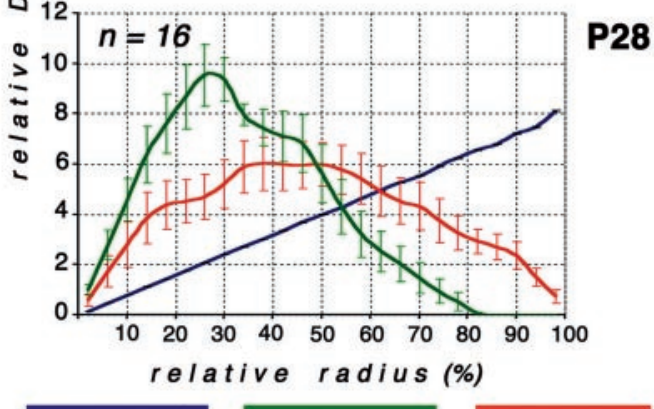

counterstain nucleoli material chromocentres

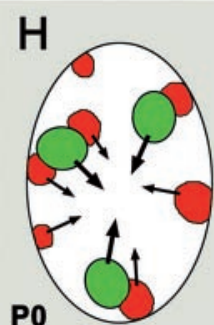

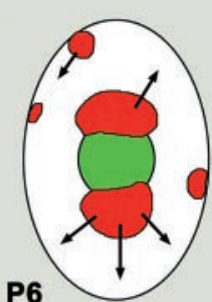

nuclei (Fig. 5A-C) to estimate the radial distribution of the nucleoli and chromocenters using the RRD (Relative Radius Distribution) computer program. Figures 5D and E demonstrate a significant shift of B23-stained nucleolar material and chromocenters from the nuclear periphery towards the nuclear center from P0 to P6. At P28 (Fig. 5F), nucleolar material showed an even more pronounced central location, while the distribution curve for the major satellite DNA signals had become broader reflecting the significant shift of pericentromeric heterochromatin back to the nuclear periphery. In accordance with the findings shown in Fig. 5D-F, the average relative radii (ARR) decreased significantly from $\mathrm{P} 0$ to $\mathrm{P} 28$ (Fig. 5G). In conclusion, compared to PC nuclei at P0, both nucleolar material and pericentromeric heterochromatin occupied more central positions at the later stages of postnatal PC development.

\section{Discussion}

Our study demonstrates changes in the number and spatial arrangement of pericentromeric heterochromatin in nuclei of postmitotic murine PCs during postnatal terminal differentiation. In the following discussion we will compare our results with the findings of previous studies and discuss biological implications.

\section{Changes of nuclear architecture during terminal PC differentiation}

Our results are consistent with the major findings of the pioneering studies performed by Manuelidis (1984b) and Martou and De Boni (2000). Martou and De Boni (2000) quantified the number of immunostained kinetochore clusters in PC nuclei and found that their average number decreased from $\mathrm{P} 0$ to $\mathrm{P} 4$, 
remained similar until P12, and increased again in stages from P15 to adults. These data correspond well with the similar decrease and increase that we observed for clusters of pericentromeric heterochromatin. It is noteworthy that the numbers for kinetochore signals reported by Martou and De Boni (2000) were higher for corresponding developmental stages than our counts of pericentromeric heterochromatin signals. This difference, apparently, reflects the fact that several clusters of kinetochores often contribute to a single chromocenter. The smaller size of immunologically detectable kinetochore signals resulted in a better spatial resolution compared to chromocenters. Accordingly, Martou and De Boni also counted higher average numbers of intermediately located kinetochore signals compared to non-adjacent chromocenters counted in the present study. An unexplained discrepancy between the results of Martou and De Boni and our study reflect the dynamic changes in the location of kinetochore clusters and chromocenters, respectively, at later stages of postnatal development. From P0 to P6 Martou and De Boni, as well as our group, consistently found a decrease of peripherally located kinetochores/chromocenters and a corresponding increase of nucleoli associated clusters. At subsequent stages (P7 to P29) and in adult mice, Martou and De Boni did not observe significant changes of the spatial cluster distribution already apparent at P5-P6. In contrast, we observed the redistribution of a fraction of pericentromeric heterochromatin clusters towards the nuclear periphery during later stages of development (P14, P21), as well as in an early adult (P28) and a 17-month-old adult.

In addition to previous studies, the present study included a quantitative assessment of dynamic changes in the position of nucleoli. For both, chromocenters and nucleoli, our data indicate movements from the nuclear periphery towards the nuclear center during the first week of postnatal development, from P0 to P6 (Fig. 5H). Centripetal movements of several nucleoli resulted in the formation of a big centrally located nucleolus (or occasionally two nucleoli), while centripetal movements of the pericentromeric heterochromatin yielded large heterochromatin clusters capping the central nucleolus.

Reduction of nucleoli number was also observed in differentiating PCs in rat and chicken (Lafarga et al., 1995). Most likely the formation of the large central nucleolus results from the fusion of several smaller nucleoli. However, the alternative possibility, i.e. that some nucleoli became inactive and disappeared, cannot be firmly excluded at present, since both silver staining or B23 staining only detect nucleoli with transcriptionally active NORs but not inactive NORs. While this question can only be settled unequivocally by FISH with NOR-specific DNA probes, we prefer the first explanation. In case that the alternative explanation were true, we should have observed the gradual disappearance of peripherally located nucleoli and the gradual growth of a centrally located nucleolus during P2, P3 and $\mathrm{P} 4$; however, this was not the case.

In addition to the obvious temporal correlation of nucleolar and heterochromatin movements, the observation that a fraction of pericentromeric heterochromatin already associated with nucleoli at P0 suggests that this fraction moves together with the nucleoli. It is not clear at present what is cause and what is effect in these coordinated centripetal movements of pericentromeric heterochromatin and nucleoli. We consider two possibilities: either heterochromatin is the primary moving structure and associated nucleoli follow or nucleoli move and associated heterochromatin follows. Heterochromatin movements may be of a primary importance since we observed that a fraction of chromocenters associated with the nuclear periphery but not with nucleoli also took part in these centripetal movements indicating that these movements occurred independently of the nucleoli. We cannot exclude, however, the possibility that other chromosomal structures than pericentromeric heterochromatin play a causal role for the observed movements. While the topology of the nucleolus almost did not change after P6, we observed a redistribution of some of the pericentromeric heterochromatin capping the nucleolus to the nuclear periphery in early (P28) and mature adults (17 months old). This movement is reflected by an increase of the average number of peripherally located chromocenters from 2 at P6 to 6.5 in adults.

Neither the mechanisms of these centripetal and centrifugal chromatin movements in postmitotic nuclei, nor the possible functional implications of such movements can be explained at present. Notably, the partial redistribution of pericentromeric heterochromatin coincides with the onset of the intense growth of the dendrite trees of PCs (Fig. 1E). Moreover, the interval between P6 and P21 is characterized by a maximum of synapse formation and synaptic activities, decreasing afterwards (due to reduction of number of spines) to the physiological level in adult animals (Weiss and Pysh, 1978). According to developmental studies of Hendelman and Aggerwal (1980), synaptic interconnections of differentiating PCs with afferents and local interneurons (basket cells and stellate cells) are greatly enhanced at P7-P10 by the formation of spines on the perisomatic region (Hendelman and Aggerwal, 1980). Enhanced synaptic input certainly means increasing physiological activity of the developing PCs involving active transcription of PC-specific genes. Martou et al. (2002) described an example of intranuclear sequence relocation correlated with onset of gene expression by showing radial relocation of the $P l c \beta 3$ gene between P3 and P5 coincident with its de novo expression between P2 and P7. Whether changes in higher-order chromatin arrangements in postmitotic PCs during the postnatal development of the cerebellum are a causal necessity or a consequence of changes of the gene expression pattern remains an intriguing question. Expression profiling data for PCs at different differentiation stages would make it possible to establish sets of DNA probes for the 3D nuclear localization of genes that become active or silent during terminal PC differentiation. Even the unequivocal demonstration of a correlation between changes in the expression status of genes and changes in their spatial nuclear and chromatin domain topology will not be sufficient per se to prove a causal relationship. A decision between cause and consequence can only be based on new approaches that allow the analysis of gene expression and silencing, respectively, after experimental manipulation of gene positions and their chromatin environment in living cells. While it seems possible to develop living cell approaches for such a purpose - e.g., using organotypic slices of cerebellum in vitro (Fenili and De Boni, 2003) - they are presently restricted to cell cultures (e.g., Tsuka- 
moto et al., 2000; Tumbar and Belmont, 2001). Such studies cannot, however, replace comprehensive studies of higherorder chromatin and gene arrangements in various cell types and in various species based on 3D multicolor FISH procedures. We anticipate that such studies will open a large field of investigations leading to an enhanced understanding of the contribution of higher-order chromatin architecture to the various epigenetic levels of gene regulation (Van Driel et al., 2003).

\section{References}

Cremer M, von Hase J, Volm T, Brero A, Kreth G, Walter J, Fischer C, Solovei I, Cremer C, Cremer $\mathrm{T}$ : Non-random radial higher-order chromatin arrangements in nuclei of diploid human cells. Chromosome Res 9:541-567 (2001).

Fenili D, De Boni U: Organotypic slices in vitro: repeated, same-cell, high-resolution tracking of nuclear and cytoplasmic fluorescent signals in live transfected cerebellar neurons by confocal microscopy. Brain Res Brain Res Protoc 11:101-110 (2003).

Hendelman WJ, Aggerwal AS: The Purkinje neuron. I. A Golgi study of its development in the mouse and in culture. J Comp Neurol 193:1063-1079 (1980).

Lafarga M, Andres MA, Fernandez-Viadero C, Villegas J, Berciano MT: Number of nucleoli and coiled bodies and distribution of fibrillar centres in differentiating Purkinje neurons of chick and rat cerebellum. Anat Embryol 191:359-367 (1995).

Legrand C, Thomasset M, Parkes CO, Clavel MC, Rabie A: Calcium-binding protein in the developing rat cerebellum. An immunocytochemical study. Cell Tissue Res 233:389-402 (1983).

\section{Acknowledgements}

We thank Joachim Walter from our group for help with the quantitative evaluation of the data.
Lentz RD, Lapham LW: Postnatal development of tetraploid DNA content in rat Purkinje cells: a quantitative cytochemical study. J Neuropathol Exp Neurol 29:43-56 (1970).

Manuelidis L: Different central nervous system cell types display distinct and nonrandom arrangements of satellite DNA sequences. Proc Natl Acad Sci USA 81:3123-3127 (1984a).

Manuelidis L: Active nucleolus organizers are precisely positioned in adult central nervous system cells but not in neuroectodermal tumor cells. J Neuropathol Exp Neurol 43:225-241 (1984b).

Manuelidis L: Indications of centromere movement during interphase and differentiation. Ann NY Acad Sci 450:205-221 (1985).

Martou G, De Boni U: Nuclear topology of murine, cerebellar Purkinje neurons: changes as a function of development. Exp Cell Res 256:131-139 (2000).

Martou G, Park PC, De Boni U: Intranuclear relocation of the Plc beta3 sequence in cerebellar Purkinje neurons: temporal association with de novo expression during development. Chromosoma 110:542-549 (2002)
Nordquist DT, Kozak CA, Orr HT: cDNA cloning and characterization of three genes uniquely expressed in cerebellum by Purkinje neurons. J Neurosci 8:4780-4789 (1988).

Tsukamoto T, Hashiguchi N, Janicki SM, Tumbar T, Belmont AS, Spector DL: Visualization of gene activity in living cells. Nat Cell Biol 2:871-878 (2000).

Tumbar T, Belmont AS: Interphase movements of a DNA chromosome region modulated by VP16 transcriptional activator. Nat Cell Biol 3:134-139 (2001).

Van Driel R, Fransz PF, Verschure PJ: The eukaryotic genome: a system regulated at different hierarchical levels. J Cell Sci 116:4067-4075 (2003).

Weiss GM, Pysh JJ: Evidence of loss of Purkinje cell dendrites during late development: a morphometric Golgi analysis in the mouse. Brain Res 154:219-230 (1978). 